\title{
Capacidad aeróbica: Actividad física musicalizada, adulto mayor, promoción de la salud Aerobic capacity: Musicalized physical activity, older adult, health promotion
}

*Anderson Aguilar Bolívar, **Jairo Alberto Flórez Villamizar, **Yanneth Saavedra Castelblanco *Secretaría de Educación de Bucaramanga (Colombia), ** Universidad Pedagógica y Tecnológica de Colombia

(Colombia)

\begin{abstract}
Resumen. Objetivo: Indagar en las diferentes bases de datos la conceptualización acerca de la definición de capacidad aeróbica, instrumentos utilizados para la medición y la relación directa con la actividad física en diferentes poblaciones en especial adulto mayor. Metodología: se realiza una búsqueda en la que se obtienen 50 artículos publicados entre los años 2013 y 2018. Esta se adelantó en bases de datos como Medline, Science Direct, Pubmed, Dialnet, Proquest, entre otras. De igual forma se consideraron aportes de expertos en el área de actividad física y promoción de la salud, donde se abordan como parámetros de búsqueda las categorías actividad física, música, capacidad aeróbica, adulto mayor. Los artículos examinados incluyeron revisiones, artículos de investigación y capítulos de libros. Resultados: al realizar el barrido bibliográfico en artículos de investigación científica y capítulos de libro, encontramos lo referente a la capacidad aeróbica, definiciones, formas de mediarla; de igual manera la relación que tiene la música al realizar actividad física y a su vez, recomendaciones para practicarla, en especial con adulto mayor. También se exponen los beneficios y riesgos de un nivel insuficiente al practicar actividad física. Así mismo, se reportan mejoras significativas en cuanto a que reduce las enfermedades, favorece el desempeño de las actividades de la vida diaria, permite ejercitar grupos musculares de piernas y brazos, logrando así realizar actividades en las cuales se usan todas las partes del cuerpo, como caminar, lo que se convierte en estrategia principal de intervención para el mantenimiento o mejora de esta capacidad y por consiguiente permita mejorar la calidad de vida. Conclusiones: Esta revisión permite que el lector visualice los diversos conceptos de capacidad aeróbica, instrumentos de medición, así como su relación con la actividad física, la música, los beneficios y recomendaciones al realizarla en distintas poblaciones, pero en especial con adulto mayor. Por otra parte, al practicar actividad física acompañada por música ha demostrado en distintas investigaciones los beneficios que otorgan al practicarlo. Aun así, se debe tener un control sobre la intensidad, como toda actividad programa, en este caso por los beats musicales (beneficios psicológicos (110-130 bpm) y físicos orgánicos (120-140 bpm), la frecuencia cardiaca, escala de Borg o test del habla. Implementar actividades novedosas como el uso de la música al realizar actividad física, crea una motivación y gusto, lo cual lleva a que los hábitos de vida cambien y mejore la calidad de vida del adulto mayor.
\end{abstract}

Palabras clave. Actividad física; capacidad aeróbica; música; adulto mayor.

\begin{abstract}
Objective: To investigate in the different databases the conceptualization about the definition of aerobic capacity, the instruments used for measurement and the direct relation with physical activity in different populations, especially older adults. Methodology: a search is carried out in which 45 articles published between 2013 and 2018 are obtained. This was advanced in databases such as Medline, Science Direct, Pubmed, Dialnet, Proquest, among others. In the same way, contributions were considered from experts in the area of physical activity and health promotion, where the following categories are addressed as search parameters: physical activity, music, aerobic capacity, and older adults. The articles examined included reviews, research articles and book chapters. Results: When we carried out the bibliographic sweep in articles of scientific research and book chapters, we found what refers to aerobic capacity, definitions, ways of mediating it; likewise, the relationship that music has when performing physical activity and at the same time, recommendations for practice, especially with older adults. It also exposes the benefits and risks of an insufficient level of physical activity. Moreover, significant improvements are reported in terms of reducing diseases, favoring the performance of activities of daily life, allowing to exercise muscle groups of legs and arms, thus achieving activities in which all parts of the body are used, such as walking, which becomes the main intervention strategy for the maintenance or improvement of this capacity and therefore allows to improve the quality of life. Conclusions: This review allows the reader to visualize the various concepts of aerobic capacity, measuring instruments, as well as their relation to physical activity, music, benefits and recommendations when performing it in different populations, but especially with older adults. On the other hand, the practice of physical activity accompanied by music has demonstrated in various investigations the benefits of practicing it. Even so, one must have control over the intensity, as any program activity, in this case by musical beats (psychological benefits (110-130 bpm) and organic physical beats (120-140 bpm), heart rate, Borg scale or speech test. Implement innovative activities such as the use of music when performing physical activity, creates a motivation and pleasure, which leads to life habits change and improve the quality of life of the older adult.
\end{abstract}

Keywords. Physical activity; aerobic capacity; music; older adults.

\section{Introducción}

Respecto a la capacidad aérobica existen varias definiciones, para (Martínez, L.E.,1985) es la capacidad para producir energía en forma permanente y prolongada a partir de los diversos nutrientes y contando claro está con un suministro adecuado de oxígeno. «la capacidad aeróbica es una

Fecha recepción: 24-09-18. Fecha de aceptación: 08-09-20

Anderson Aguilar Bolivar

aguilaranderson13@gmail.com cualidad que disminuye con la edad, $10 \%$ por década en personas sedentarias, y 15\% en edades entre 50 y 75 años» Hollenberg, Yang, Haight \& Tager (citado por Chaves-García, Sandoval-Cuellar \& Calero-Saa et al., 2017, p.1).

En este artículo se abordan los diferentes instrumentos para estimar la capacidad aeróbica en distintas poblaciones como la batería Senior fitness test por medio del test de caminata de 6 minutos (TM6M) utilizado y validado especialmente para adulto mayor en la ciudad de Tunja-Colombia (Cobo-Mejía, Ochoa, Ruiz, Vargas, Sáenz \& Sandoval Cuellar, 2016) al igual que el test de Rockport (Sandoval-Cuellar, et al., 2007) de igual forma se plantearan diferentes test utiliza- 
dos para otras poblaciones según sus necesidades o ambientes (Rosa-Guillamón, García-Cantó \& Pérez- Soto, 2017; Fajardo-López \& Moscoso-Alvarado, 2013). se incluye también dentro de la conceptualización desde autores que exponen los beneficios y recomendaciones en pro de mantener o mejorar la capacidad aeróbica (Alemán, Baranda-Andujar \& Ortín, 2014; Castillo-Retamal, León- Salgado, MondacaUrrutia, Bascuñán-Retamal \& Beltrán Bejarano, 2016).

La relación entre actividad física y la capacidad aeróbica, en la mayoría de estudios presentan aumentos significativos en la capacidad aeróbica por medio de programas de actividad física, entrenamientos desde distintas modalidades e intensidades de ejercicio en escolares (GaldamesMaliqueo, Huerta-Ojeda, Chirosa-Ríos, Cáceres-Serrano \& Reyes-Amigo, 2017; López, Martínez, Bernal, León, Garza, Almagro \& Buñuel, 2017).

La capacidad aeróbica es el componente de la condición física que está más relacionado con la salud, debido a que representa una de las cualidades más importantes de la condición física asociadas a la salud, ya que constituye una medida directa del grado general de salud y de forma específica del estado del sistema cardiovascular, metabólico y respiratorio (Valero, Ortega, Mata, Cortés, Molero \& Cuberos, 2018). Por consiguiente, tener un nivel óptimo de capacidad aeróbica disminuye el riesgo de desarrollar enfermedades cardiovasculares y aumenta la esperanza y calidad de vida en adultos mayores (Herrera, Monzó, Bartoll \& Abella, 2017; Chaves-García et al., 2017; Prieto, Del Valle, Nistal, Méndez, Abelairas-Gómez \& Barcala-Furelos, 2015).

Según Sierra (2015) la capacidad aeróbica máxima (CAM) es reconocida como el más potente indicador pronóstico de morbimortalidad cardiovascular, más preciso y fiable que la actividad física. Además, presenta una fuerte y progresiva relación inversa con la mortalidad por todas las causas y cardiovascular, en poblaciones sanas y enfermas, independiente del índice de masa corporal (IMC), genero, factores de riesgo mayores y otras comorbilidades.

La capacidad aeróbica también se asocia inversamente con distintos parámetros de salud en jóvenes, tales como composición corporal (Guillamón et al., 2014; Grao-Cruces, Nuviala \& Fernández-Martínez, 2015; Muros, Cofre-Bolados, Zurita-Ortega, Castro-Sánchez, Linares-Manrique \& ChacónCuberos, 2016; González \& Achiardi, 2016). De igual forma existe esta asociación entre la capacidad aeróbica y el rendimiento escolar (Carral, Pérez \& Espiño, 2016; Baños, Casas, Escribano, Fernández-Marcote, López \& Marcos, 2017). Para su posterior análisis, es recomendable utilizar pruebas que midan la condición física, más concretamente en los test de capacidad aeróbica, adaptados y adecuados a la edad correspondiente, dada la importancia de estudio y consecuencia para los hábitos y estilos de vida saludables de la población. Por lo tanto, entes nacionales e internacionales incluyen dentro de sus objetivos el incremento de la práctica de actividad física en todos los grupos de edad con el fin de mejorar la calidad de vida (OMS, 2018; ACSM, 2005).

De igual forma, se hace referencia a la manera en que la promoción de la actividad física se utiliza como estrategia eficiente para prevenir y tratar las enfermedades crónicas no transmisibles inherentes al sedentarismo o proceso de envejecimiento(OMS, 2010).

\section{Metodología}

Este artículo es producto del proceso de reflexión, análisis y síntesis en torno a temas relacionados con el objetivo desarrollado por la investigación «Actividad Física Musicalizada Como Alternativa Para Mejorar La Capacidad Aeróbica En El Adulto Mayor de Indeportes TunjaColombia». Para ello se elaboró un listado de interés y palabras clave dentro de las cuales se encuentra nuestro objeto de estudio «capacidad aeróbica» y otras como: actividad física, música, adulto mayor, con el fin de adelantar una búsqueda de artículos científicos en la que se obtuvieron 50 artículos publicados entre los años 2013 y 2018.

Esta búsqueda se adelantó en bases de datos como Medline, Science Direct, Pubmed, Dialnet, Proquest, entre otras. De igual forma se consideró aportes de expertos en el área de actividad física y promoción de la salud.

De igual manera se indago sobre teóricas en Torno a: Capacidad Aeróbica, Actividad Física, Música y Adulto Mayor.

\section{CapacidadAeróbica}

Se considera que la capacidad aeróbica está relacionada con la salud debido a que un bajo nivel de fitness, entendido este como una serie de hábitos equilibrados que configuran un estilo de vida saludable con un redescubrimiento en que prima la optimización de las capacidades fisiológicas y funcionales. (Corrales,2010) El cual está asociado con un riesgo incrementado de muerte prematura debido a diferentes causas, dentro de las más comunes por enfermedad cardiovascular (Sieira, 2015).

ElAmerican College of Sports Medicine (ACSM) (2005) considera la capacidad aeróbica como la capacidad para realizar un ejercicio dinámico que involucre principales grupos musculares, de intensidad alta o moderada durante periodos prolongados de tiempo. La ejecución del ejercicio depende principalmente del estado funcional de los sistemas respiratorio, locomotor y cardiovascular.

Chaves et al., (2017) de acuerdo a la investigación realizada en Tunja (Colombia) concluye que la capacidad aeróbica y la calidad de vida relacionada con la salud son condiciones que están asociadas a un declive que progresa con la edad y a estilos de vida inactivos que dependerán de valores, creencias, contexto cultural, historia personal, subjetividad, aspectos físicos, emocionales y sociales en cada una de las etapas de la vida. Por consiguiente, tiende a disminuir la capacidad aeróbica a un ritmo entre 5 a 15\% por década, luego que un individuo pasa de los 30 años de edad; estudios indican que las personas físicamente activas pueden retener una reserva suficiente de aptitud aeróbica para mantener la capacidad funcional durante sus últimos años.

En este orden de ideas cuando se habla de mejora de la aptitud física cardiorrespiratoria, depende directamente de la relación entre frecuencia, duración e intensidad del ejercicio, esta interacción entre dichos componentes prescriptivos, evidencia incrementos en el VO2 máx. oscilando entre el cinco y el $30 \%$. De acuerdo a los anteriores porcentajes aquellas personas con un nivel fitness inicial malo, incluyendo personas con cardiopatías y que muestren perdidas ostensi- 
bles de peso serán quienes muestren mayor aumento del VO2 máx. De igual forma, las personas sanas, con un nivel fitness inicial bueno, y muestran pocos cambios en su peso corporal serán las que evidenciarán aumentos menores del VO2 máx. (ACSM, 2005).

\section{Capacidad aeróbica y el rendimiento escolar}

Se ha evidenciado la asociación directa y lineal positiva entre la capacidad aeróbica durante la adolescencia y el rendimiento académico y una relación negativa débil entre obesidad y éxito académico (Baños et al., 2017). El estudio de Carral et al., (2016), realizado en 100 estudiantes que cursaban tercero, cuarto y sexto grado académico de España. Expone que los valores de capacidad aeróbica fue la capacidad que más influyó sobre la probabilidad de obtener un rendimiento académico positivo independientemente del curso académico y del género. En este mismo sentido el estudio de Gonzalez-Hernadez \& Portoles-Ariño (2016), evidencia la asociación positiva entre aspectos académicos y la práctica diaria de 30 minutos de actividad física.

En este orden de ideas, se ha evidenciado la asociación entre composición corporal y el nivel de capacidad aeróbica. El estudio realizado por Guillamón et al., (2014) con 298 escolares de educación primaria y edades comprendidas entre ocho y 12 años. Expone que los varones de mayor edad obtienen puntuaciones superiores excepto en los nueve años $(4,68)$. Por otro lado, las mujeres de $11(6,66)$ y 12 años $(6,25)$ obtuvieron puntuaciones superiores a nivel de capacidad aeróbica. Los datos del presente estudio mostraron que los escolares con un nivel superior de capacidad aeróbica presentaban una menor prevalencia de sobrepeso-obesidad. De igual forma, se asemejan los resultados de (Arriscado, Muros, Zabala \& Dalmau 2014; Palomino-Devia, GonzálezJurado \& Ramos-Parraci, 2017) donde se asocia de forma positiva una buena condición física y la composición corporal de

\section{Concepto de VO2 máximo}

El criterio de medición de la capacidad aeróbica tradicionalmente aceptado se basa en el consumo máximo de oxígeno(VO2max). La medición del VO2max comprende el análisis del consumo de oxígeno (VO2) en las muestras de aire espirado obtenidas mientras el individuo realiza un ejercicio de intensidad progresiva. Con el fin de evaluar la capacidad aeróbica, los valores del VO2 máx. se expresan normalmente en relación con el peso corporal (ml/kg/min). (ACSM, 2005, p.93), entender mejor este concepto y su medición e interpretación fisiológica.

\section{Prueba de esfuerzo máxima vs prueba de esfuerzo submáxima}

Estos dos tipos de pruebas tienen el mismo objetivo el cual es medir el VO2 máx., la decisión de escoger alguna de las dos infiere en gran medida de las razones para hacer la prueba, del tipo de persona que va a realizarla y de la disponibilidad del equipo apropiado, así como del personal.

La prueba de esfuerzo máxima por calorimetría indirecta no es un método para evaluar la capacidad aeróbica al alcance de la mayoría de personas afines en conocimientos de salud y fitness debido a sus altos costos, equipo apropiado y personal calificado. Para estos casos se cuenta con la opción de las pruebas de esfuerzo submaximas (esta prueba también requiere de la calorimetría indirecta) el cual según el ACSM (2005), el fin básico de la prueba es determinar la relación entre la frecuencia cardiaca (FC) de una persona y su VO2 durante la realización de un ejercicio progresivo, y hacer uso de esta relación para predecir el VO2 máx. Con el fin de determinar esta relación con fiabilidad, hay que medir la FC y el VO2 con dos o más intensidades de ejercicio. (p.95)

Para los dos tipos de pruebas de esfuerzo existen dos procedimientos los protocolos continuos el cual» consisten en estadios progresivos con incremento de la intensidad del trabajo sin intervalos de descanso y los protocolos discontinuos alternan intervalos de trabajo y descanso» (ACSM, 2005, p. 96). Los protocolos discontinuos presentan ventajas para algunas personas o enfermedades, en parte porque se halla con mayor frecuencia una meseta en el VO2 cuando se aumenta la intensidad del ejercicio (p. ej., el VO2 máx.), es así, que el sujeto no experimenta el grado de fatiga muscular local como ocurre en los protocolos continuos. No obstante, este tipo de protocolo tardan mucho más tiempo en realizarse y por ende, la mayor duración de estas pruebas limita su empleo y aumenta el uso de protocolos continuos en las pruebas de esfuerzo rutinarias (ACSM, 2013).

\section{Actividad Física}

La inactividad física, el sedentario, la actividad leve, ha sido uno de los factores de riesgo de mortalidad más importantes a nivel mundial, va aumentando en muchos países, lo que incide en la carga de enfermedad no transmisible y afecta directamente al estado general de salud de la población en todo el mundo. Los sujetos que no realizan suficiente ejercicio físico presentan un riesgo de mortalidad entre un $20 \%$ y un $30 \%$ superior al de aquellas que son lo suficientemente activas (OMS, 2018).

Alemán et al., (2014). Definen actividad física «Cualquier movimiento corporal producido por los músculos esqueléticos que produce un gasto energético incluye: la actividad ocupacional, práctica de deportes o ejercicio físico, tareas caseras y otras actividades» (p.12).

Para poder disminuir dichos niveles de actividad física insuficiente los países y las comunidades deben adoptar medidas que promuevan y proporcionen a las personas más oportunidades de estar activas, con el fin de incrementar la actividad física. Según la OMS (2018) las políticas destinadas a aumentar la actividad física deben estar direccionadas a lograr que en colaboración con los sectores correspondientes, se promueva la actividad física a través de acciones de la vida cotidiana; las formas activas de transporte, sean accesibles y seguras para todos; las políticas laborales y las relativas al lugar de trabajo fomenten la actividad física; en este sentido el estudio de Torres \& Jiménez, (2016), realizado en 26 oficiales de seguridad describieron la salud física y psicológica, el cual para la valoración de la capacidad aeróbica se midió con una prueba en cicloergómetro, donde se evidenció niveles de capacidad aeróbica y flexibilidad bajos, niveles de estrés mínimos, fuerza muscular promedio y personas con sobrepeso. (Avellaneda Pinzón \& Urbina 2015). 
En las empresas podemos observar cómo los empleados manifiestan mayores niveles de capacidad aeróbica o viceversa, dependiendo de las tareas laborales y la importancia que las mismas dan al fomento de actividad física en el ámbito laboral (Navarrete \& Escalona, 2017).

De igual forma, las escuelas deben contar con espacios e instalaciones seguros para que los alumnos aprovechen el tiempo libre de forma activa; las instalaciones deportivas y recreativas ofrezcan a todas las personas oportunidades para hacer deporte; y los niños reciban una educación física de calidad que les ayude a desarrollar pautas de comportamiento que los mantenga físicamente activos a lo largo de la vida (OMS, 2018).

En esta misma línea Salas- Paredes, Loreto-Montaño, Pérez- Narváez, Buela-Salazar, Canelón-Vivas \& Cortés Matheus (2016), compararon el efecto del hábito deportivo sobre la aptitud físico-motora y cardiorrespiratoria en 48 escolares con edades entre 7,5 y 9,5 años. 33 de ellos con hábito deportivo de más de seis meses con una intensidad semanal de cinco horas mínimo y 25 niños que solo realizaban las horas de actividad deportiva obligatorias escolares. Se evidenció que los escolares con hábito deportivo habitual presentan una mejor capacidad aeróbica que en los controles, mejora la aptitud físico-motora y cardiorrespiratoria sin diferencia en la capacidad funcional y pruebas bioquímicas

\section{Recomendaciones para realizar actividad física}

Las recomendaciones que se debe tener en cuenta al realizar actividad física, según la OMS (2018), señala que para personas entre edades de 5 y 17 años de edad deben al menos practicar 60 minutos de actividad física moderada o intensa, duraciones superiores procuran mayores beneficios para la salud. Deben incluirse actividades por lo menos tres veces a la semana que fortalezcan los músculos y huesos. Como se llevó a cabo en investigaciones de (Guillamón et al., 2017; Galdames-Maliqueo et al., 2017). En cambio, para adultos de 18 a 64 años de edad, lo ideal es practicar al menos 150 minutos semanales de actividad física moderada, o al menos 75 minutos semanales de actividad física intensa, para lograr mayores beneficios para la salud deben llegar a 300 minutos semanales de actividad física moderada y realizar actividades de fortalecimiento muscular 2 o más días a la semana donde se ejerciten grandes grupos musculares.

Todo individuo antes de iniciar un programa de actividad física programada debe tener en cuenta los componentes esenciales de la prescripción del ejercicio, tales como: Intensidad, duración, frecuencia y la progresión de la actividad física (OMS, 2010). Dichos componentes se aplican a individuos de todas las edades y capacidades funcionales cuando se lleva a cabo la prescripción del ejercicio, sin tener en cuenta la ausencia o presencia de factores de riesgo de enfermedades. La forma más adecuada de prescripción del ejercicio se determina mediante una evaluación objetiva de su respuesta al ejercicio, en donde se observa la respuesta de la frecuencia cardiaca (FC), la tensión arterial (TA), la escala de esfuerzo percibido (RPE), el electrocardiograma (ECG) si es posible mediante la prueba de esfuerzo progresiva. Sin embargo, no siempre se puede contar con pruebas de esfuerzo antes de iniciar un programa de ejercicio, por lo que es necesario que la prescripción del ejercicio deba hacerse pres- tando mucha atención al estado de salud del individuo incluyendo la medicación, el perfil de factores de riesgo, las características comportamentales, las preferencias respecto al ejercicio y los objetivos personales (ACSM, 2005).

La OMS recomienda realizar actividad física regular y en niveles adecuados nos ayuda a mejorar el estado osteomuscular, cardiorrespiratorio y funcional. Además; reduce el riesgo de hipertensión, accidente cerebrovascular, diabetes, cardiopatía coronaria, distintos tipos de cáncer y problemas socio -afectivos. Disminuye el riesgo de caídas y es fundamental para el equilibrio energético y el control de peso. Lo que se asemeja a los resultados dados por (Herrera et al., 2017; González \& Achiardi, 2016; Arguelles-Zayas, Infante Arguelles, Infante-Amorós, Sánchez Bruzon, CasaFigueredo, Chico-Capote \& Castell-Pérez, 2015; Sieira, 2015; Mancilla, Torres, Álvarez, Schifferli, Sapunar \& Díaz, 2014).

\section{Criterios para el control de la Intensidad}

En forma general, los métodos más utilizados para el control de la intensidad en el ejercicio aeróbico son la frecuencia cardiaca (FC) y el consumo de oxígeno (VO2). Pero presentan diferencias relevantes al momento de su control para el entrenamiento; ya que la FC se puede controlar por medio de un pulsómetro, en cambio el VO2 no se puede controlar de forma continua y simple al necesitar mediciones en laboratorio (Alemán, et al., 2014).

ElACSM (citado por Alemán et al., 2014) expone que «el margen de intensidades adecuadas para conseguir mejoras en la condición física corresponde del 40 al 85\% del VO2max» (p.23). Cuando se realiza un tipo de actividad física o entrenamiento hay características físicas evidentes que nos dan a conocer una percepción de la intensidad. Esto significa que la percepción subjetiva de la intensidad del esfuerzo (RPE) de Borg, también debe ser tenida en cuenta cuando se valora la intensidad del entrenamiento, ya que como muchos estudios demuestran relación entre la percepción subjetiva y el consumo de oxígeno (Castellanos-Fajardo \& Pulido Rull, 2009).

El ejercicio físico percibido como intenso puntúa entre 12 y 13 de la escala de Borg, el cual corresponde aproximadamente al 60\% de la FCmáx., por otra parte, el ejercicio percibido como muy intenso puntúa entre 14 al 15 y se asocia a un porcentaje cercano al 85\% de la FCmáx. Por consiguiente, la mayor parte de las personas sanas deben realizar el ejercicio dentro del rango 12 y 16, el cual corresponde entre 4 y 6 de la escala proporcional de 10 puntos (Alemán, et al., 2014).

\section{Capacidad aeróbica en adulto mayor}

La población de adulto mayor va en aumento progresivamente, «alrededor del 15\% de la población mundial es mayor de 65 años y continúa creciendo y se proyecta que las personas mayores de 65 años, que para el año 2004 eran 461 millones, pasen a ser 2 mil millones para el año 2050» (Chaves et al., 2017, p. 1).

Todos los cambios en el adulto mayor es la consecuencia de modificaciones fisiológicas que va acompañada en el proceso de envejecimiento; el ejercicio físico, si bien no evita los cambios, retrasa su aparición, disminuye su progresión y su impacto en la salud; repercutiendo en la calidad y 
estilo de vida de la persona mayor. (Jiménez Oviedo, Y., Núñez, M., \& Coto Vega, E. 2013).

Carbonel, Aparicio \& Delgado, (citado por Figueroa, Ortega, Plaza \& Vergara, 2014) señalan que al transcurrir los años, nuestra fuerza, capacidad aeróbica desciende y se reduce progresivamente la flexibilidad; además se presentan los desórdenes de equilibrio, frecuentes en el adulto mayor. Por consiguiente, conlleva a la disminución de la condición física y a la reducción de la funcionalidad.

Por su parte Castillo et al., (2016) expone que «La evidencia sobre los efectos positivos del ejercicio en varias variables asociadas al estado óptimo de salud de los adultos mayores es consistente, indicando que la cualidad base del trabajo corresponde a la capacidad aeróbica» (p.4).

En esta misma línea Hunter et al., (citado por Aguayo \& Flores, 2016) señalan que el entrenamiento aeróbico es el modo de entrenamiento más efectivo en los adultos mayores para lograr mantener y mejorar la potencia aeróbica máxima, la resistencia submaxima y la función cardiovascular. Esto conlleva a inducir «adaptaciones centrales y periféricas que mejoran el consumo máximo de oxígeno (VO2 máx.) y adaptaciones metabólicas incluyendo mayor control glicémico, aumento del aclaramiento de lípidos post prandiales, y la utilización preferencial del metabolismo oxidativo durante el ejercicio submaximo» Cadore et al., 2013; Chodzko-Zajko et al., 2009 (citado por Aguayo et al., 2016, p. 7).

En la investigación realizada por Aguayo et al., (2016) concluye que la capacidad aeróbica en adultos mayores, medida a través del TM6M o VO2max, incremento con la participación a programas de entrenamiento aeróbico, pero también con programas de entrenamiento combinado e incluso con un programa de ejercicio de resistencia

Es de resaltar la evaluación de la capacidad aeróbica en los adultos mayores. En esta revisión varios autores utilizaron el TM6M para dicha medición (Cossio-Bolaños, Selaivee, Rocha, Andruske \& Campos, 2017; Herrera et al., 2017; Cardona-García, Granada- Ramírez, Tapasco-Ypia \& TonguinoRosero, 2016; Herrera-Mor, Pablos-Monzó, Chiva-Bartoll \& Pablos-Abella, 2016; Aguayo et al., 2016; Figueroa et al., 2014) demostrando que es el test más utilizado e indicado para población adulto mayor, por su nivel de riesgo mínimo, costos bajos y confiabilidad (Cobo-Mejía et al., 2016).

Por su parte, Hall-López, Ochoa-Martínez, Alarcón-Meza, Moncada-Jiménez, Garcia-Bertruy \& Martin Dantas (2017) investigaron el efecto de un programa de entrenamiento de hidrogimnasia de 12 semanas, con frecuencia de cinco días por semana y ejercicios aeróbicos del 50\%-60\% de la FCmax sobre las capacidades físicas de 26 adultas mayores, se evidencio en la prueba de TM6M interacción significativa entre grupos y mediciones, mostrando así; la hidrogimnasia como método eficaz en la mejora de las capacidades físicas principalmente aeróbicas, de resistencia a la fuerza y agilidad de miembros inferiores.

El TM6M es otra opción válida para medir la capacidad aeróbica en pacientes con rehabilitación cardiaca y caracterizar la respuesta fisiológica en dicho test. El estudio descriptivo de González, Anchique \& Rivas, (2017), realizado en 487 individuos (hombres: 327; mujeres: 160), con edades comprendidas entre los 18 y 80 años, adscritos a un programa de rehabilitación cardiaca ubicado en altitud moderada,
(2550 metros sobre el nivel del mar), se evidencia que pacientes cardiovasculares colombianos presentan menores distancias recorridas que las demostradas en otras poblaciones y patologías. El estudio concluye que el TM6M es seguro y bien tolerado en pacientes cardiovasculares ubicados en altitud moderada, durante la prueba se evidencio cambios significativos en cuanto a respuesta cronotrópica, percepción del esfuerzo y saturación arterial de oxígeno que difieren de resultados encontrados a nivel del mar, lo cual aporta valores de referencia para las pruebas realizadas en pacientes cardiovasculares de altitud moderada.

Otra variable utilizada para medir la capacidad aeróbica es el VO2 pico (ml Kg-1 min-1), la cual fue evaluada por Fisher, McCarthy, Zuckerman, Bryan, Bickel \& Hunter (2013), con un entrenamiento combinado (aeróbico y resistencia) en distintas frecuencias semanales, el cual evidenció aumentos significativos del VO2 pico, independiente de la frecuencia semanal, de igual forma, Hunter, Bickel, Fisher, Neumeier \& McCarthy (2013) evaluó el efecto de tres frecuencias semanales diferentes de ejercicio combinado (aeróbico y resistencia) en la capacidad aeróbica de mujeres adultas mayores, medida a través del VO2 max, evidenciándose resultados similares a Fisher et al., (2013), por consiguiente, la condición física aeróbica mejoró en menor como a mayor frecuencia semanal de entrenamiento.

Por consiguiente, se puede concluir que el entrenamiento aeróbico y combinado de una frecuencia de dos veces o más por semana, puede evidenciar cambios positivos en la capacidad aeróbica. Se ha evidenciado que con una frecuencia de un día por semana con entrenamiento aeróbico (baile), durante el tiempo que sea, no presenta cambios positivos debido que la frecuencia con la que implementan la sesión de danza, no es lo suficiente para incorporar cambios significativos (Kattenstroth, Kalisch, Holt, Tegenthoff, Dinse, 2013).

Recomendaciones de actividad física en adulto mayor

Es primordial la actuación de los profesionales en la búsqueda de estrategias para que las personas mayores comprendan y reconozcan la importancia de la actividad física para la conservación de su capacidad funcional, de su independencia y de las posibilidades de interacción con otras personas.

El ejercicio aeróbico será importante ya que ayuda a mejorar la eficacia cardiaca, la capacidad respiratoria y la postura. Es importante tener en cuenta que los adultos mayores llegan a rangos aeróbicos menores que para otros grupos; es por esto que es necesario tener en cuenta la toma de frecuencia cardiaca para controlar la intensidad del ejercicio. Debido al deterioro o fragilidad ósea por la osteoporosis y a la propensión a lesiones de tendones y ligamentos, no se deben utilizar cargas muy altas en las articulaciones. En este tipo de población la coordinación se empieza a declinar, por lo tanto, se deben realizar ejercicios que puedan efectuar en forma apropiada para evitar desmotivaciones o incluso lesiones por caídas (Moreno González, A. 2005).

La actividad física debe consistir en actividades recreativas o de ocio, (paseos, caminatas, montar en bicicleta), actividades ocupacionales, es decir; cuando todavía desempeña actividad laboral, tareas domésticas, juegos, deportes o ejercicios programados en el contexto de las actividades dia- 
rias, comunitarias y familiares (OMS, 2010).

Con el propósito de mejorar las funciones cardiorrespiratorias y musculares y la salud ósea y funcional, y de reducir el riesgo de Enfermedad no transmisibles (ENT), depresión y deterioro cognitivo, la OMS (2010) recomienda que se debe practicar al menos 150 minutos semanales de actividad física moderada, o al menos 75 minutos semanales de actividad física intensa, o una combinación entre actividad moderada e intensa. Aquellos adultos mayores que tengan problemas de movilidad deben practicar actividad física para mejorar su equilibrio por lo menos 3 días a la semana; se recomienda realizar actividades de fortalecimiento muscular 2 o más días a la semana.

Las anteriores recomendaciones son válidas tanto para adultos mayores sanos de más de 65 años como para aquellos que padecen de ENT crónicas. Las personas con ciertas patologías, como enfermedades cardiovasculares y diabetes, pueden tener que tomar más precauciones y consultar a su médico antes de intentar alcanzar los niveles recomendados de actividad física para adultos mayores, siempre y cuando cada ejercicio se adapte a la capacidad funcional, de sus limitaciones y de los riesgos específicos para su salud (OMS, 2010).

«La intensidad con que se practican distintas formas de actividad física varía según las personas. Para que beneficie a la salud cardiorrespiratoria, toda actividad debe realizarse en periodos de al menos 10 minutos de duración» (OMS, 2018).

\section{Beneficios de la actividad física musicalizada en adul- tos mayores}

Entre los ejercicios físicos, como una alternativa y en beneficio de la salud en adultos mayores, se encuentra el baile, ya que posee beneficios de bienestar y aporta mejora a nivel físico, fisiológico, psicológico y emocional; en su manifestación principal, el baile puede aparecer como el resultado de una expresión emocional del ser humano Sampayo (2006, como se citó en Soljancic, 2011).

En la misma línea Heredia L (como se citó en Jiménez, et al., 2013) algunos beneficios que obtiene el adulto mayor al realizar actividad física regular encontramos mejora en aspectos sociales como: facilidad de relacionarse con los demás aumentando los contactos sociales y participación social. Aspectos físicos: incremento de la capacidad aeróbica, fuerza muscular, flexibilidad, conservar más agiles y atentos los sentidos, evita la descalcificación ósea, frena la atrofia muscular, disminuye el riesgo de enfermedades cardiovascular, favorece la movilidad articular, evita la obesidad, mejora la capacidad funcional, entre otras, evidencia que se dio en investigaciones de (Cossio-Bolaños et al., 2017; Herrera et al., 2017). Aspectos emocionales y psicológicos: mejora la capacidad de autocuidado, induce cambios positivos en el estilo de vida, disminuye la ansiedad, insomnio, depresión y contribuye en gran manera al equilibrio psicoafectivo. (Chaves et al., 2017).

La intensidad adecuada para realizar actividad física musicalizada, la cual se entiende como cualquier movimiento que genere gasto calórico y musicalizada porque es la estrategia para motivar a los participantes a realizar actividades rítmicas en las cuales se pueden controlar por medio de los beat por minuto (velocidad de la música). Por lo anterior, se ha identificado la intensidad que permite obtener beneficios psicológicos (110-130 bpm) y físico orgánicos (120-140bpm), datos que refuerzan la idea de la utilización de este medio para alcanzar, en primer término, adherencia a la práctica de actividad física y luego mejorías en los niveles de condición física y salud de las personas mayores. (Castillo et al., 2016, p. 11)

Las actividades rítmicas en el grupo de adultos mayores son comúnmente realizadas, a modo general los programas de baile aeróbico para la mejora de la condición física y cardiovascular, es recomendado como ejercicio benéfico para mantener la salud en especial de personas con bajos niveles de fitness o adultos mayores, ayudando a mantener las cualidades físicas básicas que con el proceso normal del envejecimiento van disminuyendo (Castillo et al., 2016).

Rodrigues, Krause, Farinha, Krause \& Reischak-Oliveira, (2016) en su investigación verifican los efectos del baile sobre el riesgo cardiovascular en el anciano. Estos estudios se enfocaban en resultados primarios y secundarios dentro de los cuales estaban el consumo máximo de oxígeno (VO2 pico), mediciones antropométricas y perfil lipídico. Utilizaron bases de datos para recopilar la información: MEDLINE, Cochrane Wiley, Clinical Trials.gov, bases de datos PEDRO y LILACS el cual según los criterios y calidad metodológica escogieron 7 artículos donde a pesar de las particularidades de los participantes, las características de intensidad del baile en la intervenciones siempre fue clasificada como moderada: 70\% VO 2 pico; 13-14 escala de Borg; 50-70\% de la frecuencia cardiaca máxima; 50-80\% de FRres; y el promedio de la frecuencia cardiaca entre 100 y 120 lpm. Evidenciando diferencias de medias agrupadas entre la intervención y el control mediante el modelo de efectos aleatorios. El VO2 pico mejoro en favor de bailarines $\left(3.4 \mathrm{mLkg}^{-1} \mathrm{~min}^{-1}\right.$, IC del 95\%: $1,08,5,78, \mathrm{I} 2=71 \%$ ), en comparación con los controles no ejercicio.

En este mismo sentido Cruz-Ferreira, Marmeleira, Formigo, Gomes \& Fernández (2015), investigaron el efecto de un programa de daza en la aptitud física y la satisfacción de la vida de 57 mujeres mayores (65-80) años el programa tuvo una frecuencia de tres sesiones de 50 minutos por semana durante 24 semanas, las sesiones constaban de tres fases, movilización general (15 min), fase principal (25 min), y enfriamiento (10 min). El programa fue evaluado a las 12 y 24 semanas evidenciando mejorías en la aptitud física (fuerza y la flexibilidad de los miembros inferiores, la resistencia aeróbica, agilidad motora / equilibrio dinámico, y la composición corporal) y satisfacción con la vida de las mujeres de más edad. Una duración de 12 semanas fue eficaz para mejorar la resistencia aeróbica.

\section{Conclusiones}

La capacidad aeróbica es un factor preventivo y predictivo de enfermedades crónicas no trasmisibles y el criterio de medición tradicionalmente aceptado se basa en el consumo máximo de oxígeno (VO2max) con el fin de evaluar la capacidad aeróbica, los valores del VO2 máx.

se expresan normalmente en relación con el peso corpo$\mathrm{ral}(\mathrm{ml} / \mathrm{kg} / \mathrm{min}$ ) y se vale de diferentes instrumentos para va- 
lorarla, entre ellos el más utilizado en la revisión hecha: el test de Course Navette aplicado generalmente en niños, adolescentes y adultos que presenten una salud optima y bajo consentimiento informado la aplicación de los mismos. En caso de los adultos mayores y personas sedentarias los test más utilizados dentro de las investigaciones consultadas se evidenciaron el uso de la batería Senior Fitness Test mediante el TM6M para valorar la capacidad aeróbica y el test de Rockport los cuales indicaban ser los más óptimos debido a seguridad, gastos, validez y confiabilidad.

La actividad física acompañada por música ha demostrado en distintas investigaciones los beneficios que otorgan al practicarlo. Aun así, se debe tener un control sobre la intensidad, como toda actividad programa, en este caso por los beats musicales (beneficios psicológicos (110-130 bpm) y físicos orgánicos (120-140 bpm), la frecuencia cardiaca, escala de Borg o test del habla. Implementar actividades novedosas como el uso de la música al realizar actividad física, crea una motivación y gusto, lo cual lleva a que los hábitos de vida cambien y mejore la calidad de vida del adulto mayor.

Para que haya un cambio positivo en la capacidad aeróbica del adulto mayor a través de la danza se sugiere realizarla de forma moderada: 70\% VO 2 pico; $13-14$ escala de Borg; 50-70\% de la frecuencia cardiaca máxima; 50-80\% de FRres; y el promedio de la frecuencia cardiaca entre 100 y 120 lpm.

La capacidad aeróbica, valorada por medio del TM6M o VO2max, se ve incrementada con programas de entrenamiento aeróbico, incluso por medio de entrenamientos combinado, y programas de ejercicio de resistencia, siempre y cuando se cumpla con la correcta prescripción del ejercicio para el individuo y las recomendaciones mundiales de la OMS y el Colegio Americano de Medicina del Deporte.

\section{Referencia}

American College of Sports Medicine. (2005). Guideline for exercise Testing and prescription ( $7^{\text {th }}$ edition). Badalona, España: Paidotribo.

American College of Sports Medicine. (2013). Guideline for exercise Testing and prescription (9th edition) Lippincott Williams \& Wilkins.

Aguayo, C. K., \& Flores, I. P. (2016). Efectos del entrenamiento físico sobre la capacidad física y los marcadores de la inflamación crónica en personas mayores: una revisión. Ciencias de la Actividad Física, 17(2), 71-82.

Alemán, J. A., de Baranda Andujar, P. S., \& Ortín, E. J. O. (2014). Guía para la prescripción de ejercicio físico en pacientes con riesgo cardiovascular. SEH-LELHA.

Arguelles Zayas, A. D. C., Infante Arguelles, R., Infante Amorós, A., Sánchez Bruzon, Y., Casa Figueredo, N., Chico Capote, A., \& Castell Pérez, C. (2015). Capacidad aeróbica, fuerza muscular, niveles séricos de fosfocreatincinasa y pruebas ergométricas en pacientes con polimiositis y/o dermatomiositis. Revista Cubana de Reumatología, 17(1), 6-16.

Arriscado, D., Muros, J. J., Zabala, M., \& Dalmau, J. M. (2014). Relación entre condición física y composición corporal en escolares de primaria del norte de España (Logroño). Nutrición Hospitalaria, 30(2), 385-394.

Avellaneda Pinzón, S. E., \& Urbina,A. (2015). Capacidad aeróbica de bomberos aeronáuticos. Revista de la Universidad Industrial de Santander. Salud, 47(1).

Baños, J. C. E., Casas, A. G., Escribano, L. G., Fernández-Marcote, A. R. E., López, P. T., \& Marcos, L. T. (2017). Influencia de la actividad física y la capacidad aeróbica sobre el rendimiento académico en la adolescencia: una revisión bibliográfica. Journal of Negative and No Positive Results, 3(1), 49-64.

Carral, J. M. C., Pérez, C. A., \& Espiño, M. J. S. (2016). Relación entre la condición física y rendimiento académico en matemáticas y lenguaje en estudiantes españoles de educación secundaria: Un estudio longitudinal. Cultura, Ciencia y Deporte, 11(31), 7-16.

Cardona García, N., Granada Ramírez, J. C., Tapasco Ypia, M.A., \& Tonguino-Rosero, S. (2016). Efecto de un programa de ejercicios respiratorios y aeróbicos en medio acuático versus terrestre para adultos mayores. Revista de la Universidad Industrial de Santander. Salud, 48(4).

Castellanos Fajardo, R., \& Pulido Rull, M. A. (2009). Validez y confiabilidad de la escala de esfuerzo percibido de Borg. Enseñanza e investigación en psicología, 14(1).

Castillo Retamal, M., León Salgado, M., Mondaca Urrutia, J., Bascuñán Retamal, S., \& Beltrán Bejarano, K. (2016). El ritmo y la música como herramienta de trabajo para la actividad física con el adulto mayor. Revista Ciencias de la Actividad Física $U C M, 17(1)$.

Chaves-García, M., Sandoval-Cuellar, C., \& Calero-Saa, P. (2017). Asociación entre capacidad aeróbica y calidad de vida en adultos mayores de una ciudad colombiana. Revista Peruana de Medicina Experimental y Salud Pública, 34, 672-676.

Cobo-Mejía, E. A., Ochoa, M. E., Ruiz, L. Y., Vargas, D. M., Sáenz, A. M., \& Sandoval Cuellar, C. (2016). Confiabilidad del Senior Fitness Test versión en español, para población adulta mayor en Tunja-Colombia. Archivos De Medicina Del Deporte, 33(6), 382-386.

Cossio-Bolaños, M. A., Selaivee, R. S., Rocha, C. L., Andruske, C. L., \& Campos, R. G. (2017). Capacidad funcional de adultos mayores según cambios estacionales. Nutrición clínica y dietética hospitalaria, 37(2), 83-88.

Cruz-Ferreira A, Marmeleira J, Formigo A, Gomes D, Fernandes J. (2015) Creative dance improves physical fitness and life satisfaction in older women. Res Aging. 37(8):837-855.

Fajardo-López, N., \& Moscoso-Alvarado, F. (2013). Entrenamiento de la capacidad aeróbica por medio de la terapia acuática en niños con parálisis cerebral tipo diplejía espástica. Revista de la Facultad de Medicina, 61(4), 365-371

Figueroa, Y., Ortega, A. M., Plaza, C. H., \& Vergara, M. J. (2014). Efectos de un programa de intervención en la condición física en un grupo de adultos mayores de la ciudad de Cali en 2013. Ciencia \& Salud, 2(8), 23-28.

Fisher, G., McCarthy, J., Zuckerman, P., Bryan, D., Bickel, C., \& Hunter, G. (2013).Frequency of Combined Resistance and Aerobic Training in Older Women. Journal Of Strength And Conditioning Research, 27(7), 1868-1876.

Galdames-Maliqueo, S., Huerta-Ojeda, Á., Chirosa-Ríos, L., Cáceres-Serrano, P., \& Reyes-Amigo, T. (2017). Efecto de un método de Entrenamiento Intervalado de Alta Intensidad sobre el consumo máximo de oxígeno en escolares chilenos. Universidad y Salud, 19(3), 359-365.

González, A., \& Achiardi, Ó. (2016). Relación entre capacidad aeróbica y variables antropométricas en mujeres jóvenes físicamente inactivas de la ciudad de Concepción, Chile. Revista chilena de nutrición, 43(1), 18-23.

González, N. F., Anchique, C. V., \& Rivas, A. D. (2017). Test de caminata de 6 minutos en pacientes de rehabilitación cardiaca 
de altitud moderada. Revista Colombiana de Cardiología, 24(6), 626-632.

González-Hernández, J., \& Portolés-Ariño, A. (2016). Recomendaciones de actividad física y su relación con el rendimiento académico en adolescentes de la Región de Murcia. Retos. Nuevas Tendencias en Educación Física, Deporte y Recreación, 29, 100-104

Grao-Cruces, A., Nuviala, A., \& Fernández-Martínez, A. (2015). Valoración del programa Escuelas Deportivas: composición corporal, actividad física y capacidad aeróbica en adolescentes. RETOS. Nuevas Tendencias en Educación Física, Deporte y Recreación, (27).

Guillamón, A. R., Cantó, E. G., García, P. L. R., \& Soto, J. J. P. (2014). Nivel de capacidad aeróbica y su relación con el estatus corporal en escolares de 8 a 12 años. EmásF: revista digital de educación física, (31), 7-20.

Hall López, J. A., Ochoa Martínez, P. Y., Alarcón Meza, E. I., Moncada-Jiménez, J. A., García Bertruy, O., \& Martin Dantas, E. H. (2017). Programa de entrenamiento de hidrogimnasia sobre las capacidades físicas de adultas mayores. Revista Internacional de Medicina y Ciencias de la Actividad Física y del Deporte/International Journal of Medicine and Science of Physical Activity and Sport, 17(66).

Herrera-Mor, E., Pablos-Monzó, A., Chiva Bartoll, O., \& PablosAbella, C. (2016). Efectos de un programa integral de ejercicio físico sobre la condición física, la autoestima y el grado de diversión en adultos mayores. Recuperado de http:// uvadoc.uva.es/handle/10324/23815

Herrera, E., Monzó, A. P., Bartoll, O. C., \& Abella, C. P. (2017). Efectos de la actividad física sobre la salud percibida y condición física de los adultos mayores. Journal of sport and health research, 9(1), 27-40.

Hunter G, Bickel C, Fisher G, Neumeier W, McCarthy. (2013). Combined aerobic and strength training and energy expenditure in older women. Medicine \& Science in Sports \& Exercise. 45(7), 1386-93.

Jiménez Oviedo, Y., Núñez, M., \& Coto Vega, E. (2013). La actividad física para el adulto mayor en el medio natural. InterSedes: Revista de las Sedes Regionales, 14(27).

Kattenstroth, J. C., Kalisch, T., Holt, S., Tegenthoff, M., \& Dinse, H. R. (2013). Six months of dance intervention enhances postural, sensorimotor, and cognitive performance in elderly without affecting cardio-respiratory functions. Frontiers in aging neuroscience, 5, (5).

López, J. A. H., Martínez, P. Y. O., Bernal, A. Z., León, R. S., Garza, L. G. U., Almagro, B. J., \& Buñuel, P. S. L. (2017). Efecto de un programa de actividad física de moderada a vigorosa de diez meses sobre el vo2máx y el porcentaje de grasa corporal en niños con sobrepeso y obesidad. MHSalud: Movimiento Humano y Salud, 14(1), 2.

Mancilla, R., Torres, P., Álvarez, C., Schifferli, I., Sapunar, J., \& Díaz, E. (2014). Ejercicio físico interválico de alta intensidad mejora el control glicémico y la capacidad aeróbica en pacientes con intolerancia a la glucosa. Revista médica de Chile, 142(1), 34-39.

Moreno González, A. (2005). Incidencia de la Actividad Física en el adulto mayor. Revista Internacional de Medicina y Ciencias de la Actividad Física y del Deporte/International Journal of Medicine and Science of Physical Activity and Sport, 5(20).

Muros, J. J., Cofre-Bolados, C., Zurita-Ortega, F., Castro-Sánchez, M., Linares-Manrique, M., \& Chacón-Cuberos, R. (2016). Relación entre condición física, actividad física y diferentes parámetros antropométricos en escolares de Santiago (Chile). Nutrición hospitalaria, 33(2), 314-318.
Navarrete, F. J. B., \& Escalona, E. (2017). Relación entre Capacidad Aeróbica e Índices Antropométricos en los Trabajadores Manuales Venezolanos. Revista Ingeniería Industrial, 15(2).

Organización Mundial de la Salud. (2018). Obtenido de Health Topics, Physical Activity: http://www.who.int/es/news-room/ fact-sheets/detail/physical-activity

Organización Mundial de la Salud. (2010). Recomendaciones Mundiales sobre Actividad Física para la Salud. Recuperado de: http://www.who.int/dietphysicalactivity/publications/ 9789241599979/es/

Palomino-Devia, C., González-Jurado, J. A., \& Ramos-Parraci, C. A. (2017). Composición corporal y condición física de escolares colombianos de educación secundaria y media de Ibagué. Biomédica, 37(3), 408-415.

Parra, N. S., Contreras Valencia, K., \& Castro Villamil, Á. (2012). Proceso de envejecimiento, ejercicio y fisioterapia. Revista $\mathrm{Cu}$ bana de Salud Pública, 38(4), 562-580.

Prieto, J. A., Del Valle, M., Nistal, P., Méndez, D., AbelairasGómez, C., \& Barcala-Furelos, R. (2015). Repercusión del ejercicio físico en la composición corporal y la capacidad aeróbica de adultos mayores con obesidad mediante tres modelos de intervención. Nutrición Hospitalaria, 31(3).

Rodrigues-Krause, J., Farinha, J. B., Krause, M., \& ReischakOliveira, Á. (2016). Effects of dance interventions on cardiovascular risk with ageing: Systematic review and metaanalysis. Complementary therapies in medicine, 29, 16-28. https://doi.org/10.1016/j.ctim.2016.09.004

Rosa Guillamón, A., García Cantó, E., \& Pérez Soto, J. (2017). Diferencias en la condición física en escolares de entornos rurales y urbanos de Murcia (España). REXE. Revista de Estudios y Experiencias en Educación, 16(30).

Salas Paredes, A., Loreto Montaño, I., Pérez Narváez, A., Buela Salazar, L., Canelón Vivas, E., \& Cortés Matheus, K. (2016). Hábito deportivo: efecto en la aptitud físico-motora y cardiorrespiratoria en escolares. Revista Venezolana de Endocrinología y Metabolismo, 14(2), 128-136.

Sandoval Cuellar, C., Camargo, M. L., González Ochoa, D. M., \& Vélez Ruiz, Y. P. (2007). Programa de ejercicio físico para los adultos mayores del Club Nueva Vida de la ciudad de Tunja. Revista Ciencias de la Salud, 5(2).

Sierra M. C. (2015). Relevancia actual de la capacidad aeróbica máxima en la práctica clínica. Archivos de medicina del deporte: revista de la Federación Española de Medicina del Deporte y de la Confederación Iberoamericana de Medicina del Deporte, (168), 239-247.

Soljancic, M. G. B. (2011). Influencia del baile recreativo en el estado de ánimo y autoestima de personas adultas. Eureka (Asunción) en Línea, 8(2), 241-253.

Torres, J. M. B., \& Jiménez, J. M. (2016). Salud física y estrés en oficiales de seguridad de la Universidad de Costa Rica en el año 2014. Rev. Costa rica. Salud pública, 25(1), 8-17.

Valero, G. G., Ortega, F. Z., Mata, S. S. R., Cortés, A. J. P., Molero, P. P., \& Cuberos, R. C. Análisis de la capacidad aeróbica como cualidad esencial de la condición física de los estudiantes: Una revisión sistemática (Analysis of aerobic capacity as an essential quality of students' physical condition: A systematic review). Retos, (34), 395-402. 